\title{
Design of Optical Wireless Networks with Fair Traffic Flows
}

\author{
Artur Tomaszewski \\ Institute of Telecommunications, Warsaw University of Technology, Poland \\ Correspondence should be addressed to Artur Tomaszewski; artur@tele.pw.edu.pl
}

Received 7 March 2014; Accepted 7 May 2014; Published 30 June 2014

Academic Editor: Dritan Nace

Copyright (C) 2014 Artur Tomaszewski. This is an open access article distributed under the Creative Commons Attribution License, which permits unrestricted use, distribution, and reproduction in any medium, provided the original work is properly cited.

\begin{abstract}
The paper presents a method for optimising the wireless optical network that carries elastic packet traffic. The particular focus is on modelling the effect of elastic traffic flows slowing down in response to the decrease of the optical transmission systems' capacity at bad weather conditions. A mathematical programming model of the network design problem is presented that assumes that the packet rates of elastic traffic flows decrease fairly. While practically any subset of network links can be simultaneously affected by unfavourable transmission conditions, a particular challenge of solving the problem results from a huge number of network states considered in the model. Therefore, how the problem can be solved by generating the most unfavourable network states is presented. Moreover, it is proved that it is entirely sufficient to consider only the states that correspond to the decrease of capacity on a single link. Finally, as the general problem is nonlinear, it is shown that the problem can be transformed to a linear MIP problem and solved effectively when single-path routing of traffic flows is assumed.
\end{abstract}

\section{Problem Definition}

The paper considers the problem of designing a wireless optical network that carries elastic packet traffic. Each node of the considered network is a packet router, while each link connecting a pair of nodes is a packet link composed of a number of optical wireless transmission systems (cf. Figure 1).

Employing optical wireless transmission systems has many advantages, the major one being that little network infrastructure is required. Thus, the network can be installed or expanded quickly and the installation process is comparatively inexpensive. The network is also very flexible as far as reconfiguration is concerned-a node can be easily reconnected to a different set of nodes and the transmission equipment can be deinstalled and moved to another location quickly. However, there are also disadvantages. For example, the transmission systems are line-of-sight systems, which means that the optical transmitter must see the optical receiver. That limits the feasible network configurations and may heavily influence the network design as shown in [1].

The most important issue, however, is that the transmitted signal is vulnerable to atmospheric conditions (smoke, fog, smog, etc.) as the systems are wireless and no transmission medium is used (cf. [2]). The worsening of atmospheric conditions may heavily influence the quality of the received optical signal. With that in mind, the optical wireless transmission systems are designed to operate in a number of transmission modes. Based on a specific scheme of signal coding and modulation, each mode provides particular capacity of the transmission system and particular robustness of the optical signal to unfavourable transmission conditions-in general, the higher the system's bit-rate the lower the signal's robustness. Thus, using multiple schemes effectively solves the trade-off between signal robustness and system capacity.

At perfect weather conditions the transmission systems of the wireless optical network are supposed to operate using the highest-capacity transmission mode, which provides the maximum capacity of links. But when the signal propagation conditions deteriorate, the operating mode of each affected transmission system changes to a more robust one and the capacity of network links decreases. Whenever the resulting capacity of a network link is less than the total nominal bandwidth of the information flows assigned to the link, packet losses are inevitable.

With elastic packet traffic, packet losses will cause packet sources to adapt (lower) their packet rate to match the available network bandwidth. This behaviour is due to the 


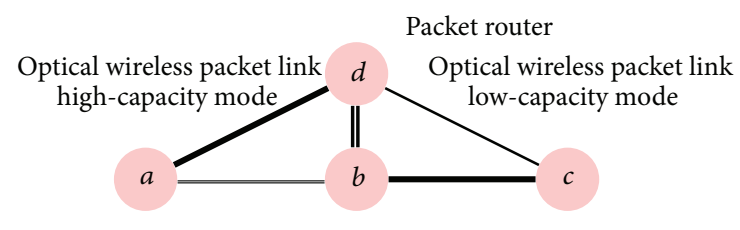

FIGURE 1: An optical wireless network.

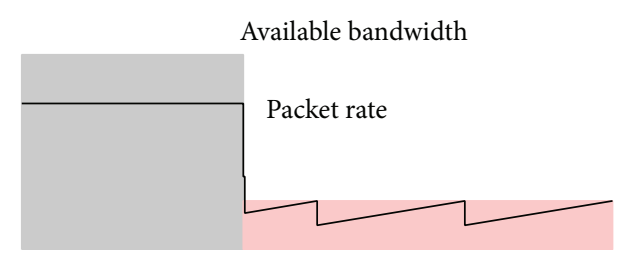

Figure 2: Elastic traffic behaviour. per session end-to-end flow control mechanisms of the TCP data transport protocol. The packet rate of the TCP session is increased linearly every time the sender application receives an acknowledgement that a packet has reached the destination, and the rate is decreased geometrically every time a packet is lost (cf. Figure 2). Since the packet losses happen randomly, arguably, the decrease of the packet rates of the sessions on a given link must be fair, meaning that the sessions with higher packet rates decrease the rate first and all sessions decrease the rate to the same value.

Changing the rate at which packets are sent directly affects the perceived quality of service and should be controlled by careful network design. The network design problem considered in this paper consists in dimensioning the links of a given optical wireless network and routing a given set of elastic traffic flows so that the total cost of links (i.e., the total cost of the optical wireless transmission systems installed on the links) does not exceed a given budget $B>0$, and in nonnominal transmission conditions the bandwidth that is assigned to any single traffic session is decreased by at most a given factor $\gamma, 0<\gamma<1$. Alternatively, the objective of the design may be to minimise the total cost of links or to maximise the minimal bandwidth reduction. It is assumed that any subset of network links can be affected by unfavourable transmission conditions and that the decrease of packet rates on an affected link is fair.

The considered problem is similar to the classical problems of survivable network design (cf. [3]). However, there are a couple of major differences. First, in this paper a max-min fair distribution of traffic is considered instead of the general one; moreover, the fairness is considered at the level of individual sessions and not aggregated traffic flows. The application of the max-min fairness concept in network design and the approaches to solving max-min fairness problems are discussed in [4, 5]. Second, designing survivable networks is based on the notion of the set of network states, which usually consists of the nominal (failurefree) state and a number of nonnominal (failure) states. And the particular issue considered in this paper (apart from the fact that links do not fail but their capacity is decreased instead) is that in the case of the optical wireless network no meaningful set of nonnominal network states can be defined (what is the extent of fog?) - actually, any subset of links can be affected by unfavourable transmission conditions, which leads to a huge number of network states. Dealing with that issue is a major problem considered in this paper.

\section{Problem Modelling}

Let the optical wireless network be modelled with a directed graph $\mathscr{G}=(\mathscr{V}, \mathscr{E})$, with $\mathscr{V}$ being the set of nodes and $\mathscr{E}$ being the set of links, and let $\mathscr{P}$ be the set of paths in $\mathscr{G}$. For each $p \in \mathscr{P}$, let $E_{P}(e) \subseteq \mathscr{E}$ denote the set of links of path $p$, and, for each $e \in \mathscr{E}$, let $P_{E}(e) \subseteq \mathscr{P}$ denote the set of paths that use linke.

Let the links of the network be realised with one type of transmission system that can operate in two transmission modes-the primary (high-capacity) mode which is used in nominal transmission conditions and the secondary (lowcapacity) mode which is used when conditions deteriorate. Without loss of generality it can be assumed that the capacity of the system is equal to 1 in the high-capacity mode and is equal to $\alpha, 0<\alpha<1$, in the low-capacity mode. Each link of the network can be equipped with a number of transmission systems. Let the link cost function $\xi: \mathscr{E} \mapsto \mathbb{R}_{+}$define the cost of a single transmission system installed on the link.

Let $\mathcal{S}$ denote the set of network states. Let $s_{0}$ denote the nominal network state of perfect transmission conditions (when all transmission systems operate in the high-capacity mode) and let set $\mathcal{S}_{f}$ of nonnominal network states represent all assumed situations of unfavourable transmission conditions; thus, $\mathcal{S} \equiv\left\{s_{0}\right\} \cup \mathcal{S}_{f}$. In particular, $\mathcal{S}_{f}$ could be defined by assuming that at most $N$ links of the network can be simultaneously affected by unfavourable signal transmission conditions. For each $s \in \mathcal{S}_{f}$, let $E_{S}(s) \subseteq \mathscr{E}$ be the set of affected links in nonnominal state $s$. It is assumed that whenever transmission conditions on a network link deteriorate, all the transmission systems installed on the link operate in the low-capacity transmission mode.

Let the network traffic be modelled with a set of demands $\mathscr{D}$. Let function $s: \mathscr{D} \mapsto \mathbb{Z}_{+}$define the number of sessions that correspond to the demand, function $r: \mathscr{D} \mapsto \mathbb{R}_{+}$define the nominal packet rate of a single session, and function $l$ : $\mathscr{D} \mapsto \mathbb{R}_{+}$define the average packet length for the demand (it can be noticed that $b(d) \equiv r(d) l(d)$ is the nominal bandwidth required by a single session and $h(d) \equiv r(d) l(d) s(d)$ is the volume of demand $d \in \mathscr{D})$. Finally, for each $d \in \mathscr{D}$, let $\mathscr{P}(d) \subseteq \mathscr{P}$ denote the set of admissible paths of demand $d$.

For each $e \in \mathscr{E}$, let variable $y_{e}$ denote the number of transmission systems installed on link $e$. For each $d \in \mathscr{D}$ and $p \in \mathscr{P}(d)$, let variable $x_{d p}$ denote the number of sessions of demand $d$ assigned to path $p$, and, for each $d \in \mathscr{D}, p \in \mathscr{P}(d)$, and $s \in \mathcal{S}_{f}$ (it could be assumed that $E_{P}(p) \cap E_{S}(s) \neq \emptyset$ ), let variable $z_{d p s}$ denote the reduction of the packet rate of the sessions of demand $d$ assigned to path $p$ in state $s$ (if $z_{d p s}$ equals 1 , the packet rate is not reduced). The network 
design problem can now be formulated as a mathematical programme with the following constraints:

$$
\begin{gathered}
\sum_{e \in \mathscr{E}} \xi(e) y_{e} \leq B \\
\sum_{p \in \mathscr{P}(d)} x_{d p}=s(d) \quad d \in \mathscr{D} \\
\sum_{d \in \mathscr{D} p \in \mathscr{P}(d) \cap P_{E}(e)} r(d) l(d) x_{d p} \leq y_{e} \quad e \in \mathscr{E} \\
\sum_{d \in \mathscr{D} p \in \mathscr{P}(d) \cap P_{E}(e)} r(d) l(d) x_{d p} z_{d p s} \leq \alpha y_{e} \quad s \in \mathcal{S}_{f}, e \in E_{S}(\text { (l) } \\
\left.\gamma \leq z_{d p s} \leq 1 \quad \text { (1) }\right) \\
x_{d p} \in \mathbb{Z}_{+} \quad d \in \mathscr{D}, p \in \mathscr{P}(d) \\
y_{e} \in \mathbb{Z}_{+} \quad e \in \mathscr{E} .
\end{gathered}
$$

Let $y \equiv\left(y_{e}\right)_{e \in \mathscr{E}}, x \equiv\left(x_{d p}\right)_{d \in \mathscr{D}, p \in \mathscr{P}(d)}$, and $z \equiv$ $\left(z_{d p s}\right)_{d \in \mathscr{D}, p \in \mathscr{P}(d), s \in \mathcal{S}_{f}}$. Let $\mathscr{W}\left(B, \gamma, \mathcal{S}_{f}\right)$ denote the feasibility set defined by constraints (1a), (1b), (1c), (1d), (1e), (1f), and $(\mathrm{lg})$ being the set of triples $(y, x, z)$. Seemingly, to complete the formulation of the network design problem, additional constraints are still required to express the conditions that must be satisfied by variables $z_{d p s}$; the constraints should reflect the effect of fairly slowing down the elastic traffic sessions in response to the decrease of link capacities in nonnominal states $s \in \mathcal{S}_{f}$. That effect can be modelled as follows.

Consider a given nonnominal state $s \in \mathcal{S}_{f}$. Consider vector $Z \equiv z_{s}$. Let $\mathscr{F}$ denote the set of affected network links, that is, $\mathscr{F} \equiv E_{S}(s)$, and let $c$ be the vector of their capacities: $c \equiv\left(c_{e} \in \mathbb{R}_{+}: e \in \mathscr{F}, c_{e}=\alpha y_{e}\right)$ (i.e., $\left.\left.c \equiv \alpha y\right|_{E_{S}(s)}\right)$. For each $d \in$ $\mathscr{D}, p \in \mathscr{P}(d)$, and $e \in E_{p}(p) \cap \mathscr{F}$, let $t_{d p e}$ be a binary variable that equals 1 if the decrease of capacity of link $e$ causes the decrease of the packet rate of the sessions of demand $d$ that are routed along path $p$ and 0 otherwise. For each $e \in \mathscr{F}$, let $u_{e}$ be a binary variable that equals 1 if link $e$ is saturated (i.e., the total flow $\sum_{d \in \mathscr{D}} \sum_{p \in \mathscr{P}(d) \cap P_{E}(e)} r(d) l(d) x_{d p} z_{d p s}$ on the link is equal to the link's capacity) and 0 otherwise; the decrease of capacity of a link can cause the decrease of a session's packet rate only if the link becomes saturated. For each $e \in \mathscr{F}$, let variable $v_{e}$ denote the common packet rate of the sessions that have their rate reduced due to the decrease of capacity of link $e$; $v_{e}$ is also the maximum session packet rate on link $e$. The condition for packet rate reduction can be expressed as follows:

$$
Z_{d p}=\min \left\{1, \min _{e \in E_{P}(p) \cap \mathscr{F}} \frac{v_{e}}{r(d)}\right\} \quad d \in \mathscr{D}, p \in \mathscr{P}(d) .
$$

Altogether, the following constraints must hold ( $C$ and $R$ are sufficiently large constants, e.g., $C \equiv \sum_{d \in \mathscr{D}} h(d)$ and $\left.R \equiv \max _{d \in \mathscr{D}} r(d)\right)$ which express the relation between packet rate reduction and capacity decrease of links from $\mathscr{F}$ (the constraints are based on the model of the max-min fair network flows proposed in [6]):

$$
\begin{aligned}
& 1-Z_{d p} \leq \sum_{e \in E_{P}(p) \cap \mathscr{F}} t_{d p e} d \in \mathscr{D}, p \in \mathscr{P}(d) \\
& t_{d p e} \leq u_{e} \quad d \in \mathscr{D}, p \in \mathscr{P}(d), e \in E_{P}(p) \cap \mathscr{F} \\
& 0 \leq c_{e}-\sum_{d \in \mathscr{D} p} \sum_{p \in \mathscr{P}(d) \cap P_{E}(e)} r(d) l(d) x_{d p} Z_{d p} \leq C\left(1-u_{e}\right) \\
& e \in \mathscr{F} \\
& 0 \leq v_{e}-r(d) Z_{d p} \leq R\left(1-t_{d p e}\right) \quad d \in \mathscr{D}, p \in \mathscr{P}(d), \\
& e \in E_{P}(p) \cap \mathscr{F} \\
& R\left(1-u_{e}\right) \leq v_{e} \leq R \quad e \in \mathscr{F} \\
& u_{e} \in\{0,1\} \quad e \in \mathscr{F} \\
& t_{d p e} \in\{0,1\} \quad d \in \mathscr{D}, p \in \mathscr{P}(d), e \in E_{P}(p) \cap \mathscr{F} \\
& 0 \leq Z_{d p} \leq 1 \quad d \in \mathscr{D}, p \in \mathscr{P}(d) .
\end{aligned}
$$

Let $Q(c, x, \mathscr{F})$ denote the feasible set defined by constraints (3a), (3b), (3c), (3d), (3e), (3f), (3g), and (3h), and let $Q_{Z}(c, x, \mathscr{F})$ denote the projection of $Q(c, x, \mathscr{F})$ onto the set of vectors $Z$. It can be shown that set $Q(c, x, \mathscr{F})$ is nonempty. Actually, for a vector $Z$ to satisfy constraints (3a), (3b), (3c), (3d), (3e), (3f), (3g), and (3h), vector $\left(r(d) Z_{d p}: d \in \mathscr{D}, p \in\right.$ $\mathscr{P}(d), x_{d p}>0$ ) must be max-min fair. Thus finding vector $Z \in$ $Q_{Z}(c, x, \mathscr{F})$ can be formulated as a max-min fair optimisation problem having vector $Z \equiv 0$ as a feasible solution.

Assuming the objective of minimising the total cost of links, the considered network design problem, denoted by $P\left(\gamma, \mathcal{\delta}_{f}\right)$, can now be defined as follows:

$$
\begin{aligned}
P\left(\gamma, \mathcal{S}_{f}\right): \quad & \min B \\
& (y, x, z) \in \mathscr{W}\left(B, \gamma, \mathcal{\delta}_{f}\right) \\
& z_{s} \in Q_{Z}\left(\left.\alpha y\right|_{E_{S}(s)}, x, E_{S}(s)\right) \quad s \in \mathcal{S}_{f} \\
& B \geq 0 .
\end{aligned}
$$

\section{Problem Solving}

Potentially, the number of nonnominal network states is huge as each subset of links $\mathscr{F} \subseteq \mathscr{E}$ can correspond to a nonnominal network state. Thus, in general, the number of constraints and variables of problem (4a), (4b), (4c), and (4d) is also huge. Still, problem (4a), (4b), (4c), and (4d) can be solved with the constraint and column generation approach, by generating nonnominal network states (and thus the constraints and variables that correspond to those states) on the "as-needed" basis.

Problem (4a), (4b), (4c), and (4d) defined with a restricted set $\mathcal{S}_{f}$ of nonnominal states can be treated as the 
master problem. Let $\left(y^{*}, x^{*}, z^{*}\right)$ be an optimal solution to the master problem. For given $y^{*}$ and $x^{*}$, the slave problem consists in finding such set of links affected by unfavourable signal transmission conditions that for at least one path of a demand the reduction of the packet rate is less than $\gamma$; this set corresponds to a new nonnominal network state $s^{*}$, which is then added to set $\mathcal{S}_{f}$ of the master problem.

For each $e \in \mathscr{E}$, let $q_{e}$ be a binary variable that equals 1 if link $e$ belongs to the required set $E_{S}\left(s^{*}\right)$ and 0 otherwise. For each $d \in \mathscr{D}$ and $p \in \mathscr{P}(d)$, let variable $Z_{d p}$ denote the reduction of the packet rate of the sessions of demand $d$ assigned to path $p$ in the required state, and let $Z \equiv$ $\left(Z_{d p}\right)_{d \in \mathscr{D}, p \in \mathscr{P}(d)}$. Let $\theta \equiv \min _{d \in \mathscr{D}, p \in \mathscr{P}(d)} Z_{d p}$, and for each $d \in \mathscr{D}$ and $p \in \mathscr{P}(d)$, let $r_{d p}$ be a binary variable that equals 1 whenever $Z_{d p}=\theta$. Then, a new nonnominal network state can be found by solving the following slave problem $R(y, x)$ for $y^{*}$ and $x^{*}$ which minimises the value of $\theta$ over all sets $\mathscr{F} \subseteq \mathscr{E}$ (it could also be assumed that $|\mathscr{F}| \leq N$ ):

$$
\begin{aligned}
R(y, x): \quad & \min \theta \\
& 0 \leq Z_{d p}-\theta \leq 1-r_{d p} \quad d \in \mathscr{D}, p \in \mathscr{P}(d) \\
& \sum_{d \in \mathscr{D}, p \in \mathscr{P}(d)} r_{d p} \geq 1 \\
& c_{e}=y_{e}-q_{e}(1-\alpha) y_{e} \quad e \in \mathscr{E} \\
& Z \in Q_{Z}(c, x, \mathscr{E}) \\
& c_{e} \geq 0 \quad e \in \mathscr{E} \\
& q_{e} \in\{0,1\} \quad e \in \mathscr{E} \\
& r_{d p} \in\{0,1\} \quad d \in \mathscr{D}, p \in \mathscr{P}(d) \\
& 0 \leq Z_{d p} \leq 1 \quad d \in \mathscr{D}, p \in \mathscr{P}(d) \\
& 0 \leq \theta \leq 1 .
\end{aligned}
$$

It can be noticed that constraints (3a), (3b), (3c), (3d), (3e), (3f), (3g), and (3h) defining set $Q_{Z}(c, x, \mathscr{E})$ can be directly embedded into the formulation of $R(y, x)$.

If the optimal objective value $\theta^{*}$ of slave problem $R\left(y^{*}, x^{*}\right)$ is greater or equal to $\gamma$, the optimal solution of the master problem is the optimal solution of the considered network design problem. Otherwise, the master problem must be modified by adding variables and constraints that correspond to a new nonnominal network state $s^{*} \in \mathcal{S}_{f}$ : the optimal solution vector $q^{*} \equiv\left(q_{e}^{*}\right)_{e \in \mathscr{E}}$ of problem $(5 \mathrm{a})$, (5b), (5c), (5d), (5e), (5f), (5g), (5h), (5i), and (5j) defines the values of the characteristic function of set $E_{S}\left(s^{*}\right)$; that is, $E_{S}\left(s^{*}\right) \equiv\left\{e \in \mathscr{E}: q_{e}^{*}=1\right\}$.

Solving problem (4a), (4b), (4c), and (4d) with state generation approach does not guarantee that considering a large number of network states can be avoided. However, careful examination of the packet rate reduction condition (2) leads to a conclusion that, actually, it is sufficient to consider only single-link states in the design problem. This fact can be expressed formally with the following proposition.
Proposition 1. Consider an arbitrary set of nonnominal network states $\mathcal{S}_{f}$ and set $\widehat{\mathcal{S}}_{f}$ such that $\left|E_{S}(s)\right|=1$ for each $s \in \widehat{\mathcal{S}}_{f}$ and $\bigcup_{s \in \mathcal{S}_{f}} E_{S}(s)=\bigcup_{s \in \hat{\mathcal{S}}_{f}} E_{S}(s)$. If $\left(y^{*}, x^{*}, z^{*}\right)$ is a feasible solution of problem $P\left(\gamma, \widehat{\mathcal{S}}_{f}\right)$, then there exists a feasible solution $\left(y^{*}, x^{*}, z\right)$ of problem $P\left(\gamma, \mathcal{S}_{f}\right)$.

Proof. Consider a set of vectors $z_{s}, s \in \mathcal{S}_{f}$, such that $z_{s} \in$ $Q_{Z}\left(\left.\alpha y^{*}\right|_{E_{S}(s)}, x^{*}, E_{S}(s)\right)$ (recall that sets $Q_{Z}$ are not empty). It will be proved that $\left(y^{*}, x^{*}, z\right)$ is a feasible solution of problem $P\left(\gamma, \mathcal{S}_{f}\right)$.

Due to (4a), (4b), (4c), and (4d), it must be shown that $\left(y^{*}, x^{*}, z\right) \in \mathscr{W}\left(B, \gamma, \mathcal{S}_{f}\right)$. As $\left(y^{*}, x^{*}, z^{*}\right)$ is a feasible solution of problem $P\left(\gamma, \widehat{\mathcal{S}}_{f}\right)$, therefore $\left(y^{*}, x^{*}, z^{*}\right) \in \mathscr{W}\left(B, \gamma, \mathcal{S}_{f}\right)$, and it is sufficient to prove that vector $z$ satisfies constraints (1e); that is, $z_{d p s} \geq \gamma$ for each $d \in \mathscr{D}, p \in \mathscr{P}(d), s \in \mathcal{S}_{f}$.

Assume that there exist $d^{\prime} \in \mathscr{D}, p^{\prime} \in \mathscr{P}\left(d^{\prime}\right)$, and $s^{\prime} \in \mathcal{S}_{f}$, such that $z_{d^{\prime} p^{\prime} s^{\prime}}<\gamma$. Due to (2), there must exist link $e^{\prime} \in E_{P}(p) \cap E_{S}\left(s^{\prime}\right)$ such that $z_{d^{\prime} p^{\prime} s^{\prime}}=v_{e^{\prime}}^{\prime} / r\left(d^{\prime}\right)$ for $Q\left(\left.\alpha y^{*}\right|_{E_{S}\left(s^{\prime}\right)}, x^{*}, E_{S}\left(s^{\prime}\right)\right)$; obviously, that link must be saturated in $s^{\prime}$; that is, $\sum_{d \in \mathscr{D}} \sum_{p \in \mathscr{P}(d) \cap P_{E}\left(e^{\prime}\right)} r(d) l(d) x_{d p} z_{d p s^{\prime}}=\alpha y_{e^{\prime}}^{*}$. According to the assumption, there exists state $\widehat{s} \in \widehat{\delta}_{f}$, such that $E_{S}(\widehat{s})=\left\{e^{\prime}\right\}$. With $\left(y^{*}, x^{*}, z^{*}\right)$ being a feasible solution of problem $P\left(\gamma, \widehat{S}_{f}\right)$, due to (4a), (4b), (4c), and (4d), $z_{\widehat{s}}^{*} \in Q_{Z}\left(\left.\alpha y^{*}\right|_{E_{S}(\widehat{s})}, x^{*}, E_{S}(\widehat{s})\right)$ and $z_{d p \widehat{s}}^{*} \geq \gamma$ for each $d \in \mathscr{D}$ and $p \in \mathscr{P}(d)$, in particular, $z_{d^{\prime} p^{\prime} \hat{s}}^{*} \geq \gamma$. Therefore, due to (2), $\widehat{v}_{e^{\prime}} / r\left(d^{\prime}\right) \geq \gamma$ for $Q\left(\left.\alpha y^{*}\right|_{E_{S}(\widehat{s})}, x^{*}, E_{S}(\widehat{s})\right)$, and thus $\widehat{v}_{e^{\prime}}>v_{e^{\prime}}^{\prime}$. Then, once again due to (2) (and the fact that $\mathscr{F}=$ $\left.E_{S}(\widehat{s})=\left\{e^{\prime}\right\}\right), z_{d p \widehat{s}}^{*}=\min \left\{1, \widehat{v}_{e^{\prime}} / r(d)\right\} \geq \min \left\{1, v_{e^{\prime}}^{\prime} / r(d)\right\} \geq$ $z_{d p s^{\prime}}$ for each $d \in \mathscr{D}, p \in \mathscr{P}(d) \cap P_{E}\left(e^{\prime}\right)$, and $z_{d^{\prime} p^{\prime} \widehat{s}}^{*}>z_{d^{\prime} p^{\prime} s^{\prime}}$. Thus, $\sum_{d \in \mathscr{D}} \sum_{p \in \mathscr{P}(d) \cap P_{E}\left(e^{\prime}\right)} r(d) l(d) x_{d p} z_{d p \widehat{s}}^{*}>$ $\sum_{d \in \mathscr{D}} \sum_{p \in \mathscr{P}(d) \cap P_{E}\left(e^{\prime}\right)} r(d) l(d) x_{d p} z_{d p s^{\prime}}=\alpha y_{e^{\prime}}^{*}$, which is not possible.

The intuition behind the proof of Proposition 1 is that the packet rate on any given link decreases most if that link is the only overloaded link in the network; decreasing the capacity of other links can only decrease the load on that particular link and minimise the need for packet rate decrease. Consequently, the opposite of Proposition 1 is not true as stated by the following proposition.

Proposition 2. Consider an arbitrary set of nonnominal network states $\mathcal{S}_{f}$ and set $\widehat{\mathcal{S}}_{f}$ such that $\left|E_{S}(s)\right|=1$ for each $s \in \widehat{\mathcal{S}}_{f}$ and $\bigcup_{s \in \mathcal{S}_{f}} E_{S}(s)=\bigcup_{s \in \widehat{\mathcal{S}}_{f}} E_{S}(s)$. If $\left(y^{*}, x^{*}, z^{*}\right)$ is a feasible solution of problem $P\left(\gamma, \mathcal{S}_{f}\right)$, then there need not exist a feasible solution $\left(y^{*}, x^{*}, z\right)$ of problem $P\left(\gamma, \widehat{\delta}_{f}\right)$.

Proof. Consider a network illustrated in Figure 3, which consists of nodes $a, b$ and $c$, links $e_{1} \equiv(a, b)$ and $e_{2} \equiv(b, c)$, and demands $d_{1}$ from $a$ to $b$ and $d_{2}$ from $a$ to $c$, such that $h\left(d_{1}\right)=1 / 2, r\left(d_{1}\right)=1$ and $h\left(d_{2}\right)=1, r\left(d_{2}\right)=1 / 2$. Let $\mathcal{S}_{f} \equiv\{s\}$, such that $E_{S}(s) \equiv\left\{e_{1}, e_{2}\right\}$. Let $\alpha=1 / 2$ and $\gamma=1 / 2$. Consider a solution to problem $P\left(\gamma, \mathcal{S}_{f}\right)$ (obviously, it is the optimal solution), such that $y_{e_{1}}^{*}=2$ and $y_{e_{2}}^{*}=1$ 


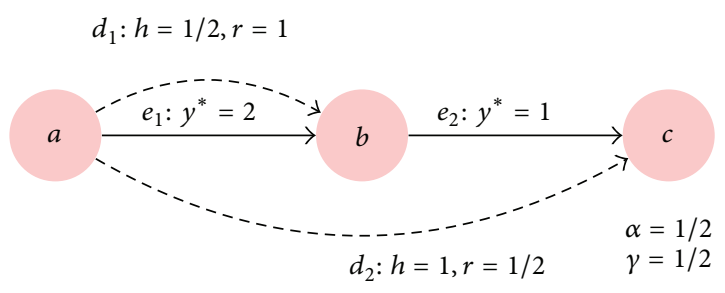

FIgURE 3: A network example for infeasibility of problem $P\left(\gamma, \widehat{\delta}_{f}\right)$.

and $x_{d_{1}}^{*}=s\left(d_{1}\right)$ and $x_{d_{2}}^{*}=s\left(d_{2}\right)$ (index $p$ is skipped as there is only one path for each demand): it can be checked that $z_{d_{1} s}^{*}=1$ and $z_{d_{2} s}^{*}=1 / 2$ (again with index $p$ skipped). However, for state $\widehat{s}$, such that $E_{S}(\widehat{s}) \equiv\left\{e_{1}\right\}$, link $e_{1}$ is saturated since $h\left(d_{1}\right)+h\left(d_{2}\right)>\alpha y_{e_{1}}^{*}=1$, and inevitably the packet rates of both flows must decrease as $r\left(d_{1}\right)>r\left(d_{2}\right)$ and $h\left(d_{2}\right)=\alpha y_{e_{1}}^{*}$. Thus, $s\left(d_{1}\right) l\left(d_{1}\right) v_{\widehat{s} e_{1}}+s\left(d_{2}\right) l\left(d_{2}\right) v_{\widehat{s}_{1}}=\alpha y_{e_{1}}^{*}=1$ and $v_{\hat{s} e_{1}}=1 /\left(h\left(d_{1}\right) / r\left(d_{1}\right)+h\left(d_{2}\right) / r\left(d_{2}\right)\right)=2 / 5$, and then $z_{d_{1} \widehat{s}}=v_{\widehat{s} e_{1}} / r\left(d_{1}\right)=2 / 5<\gamma$.

Proposition 1 leads directly to the following conclusion.

Corollary 3. Consider an arbitrary set of nonnominal network states $\mathcal{S}_{f}$ and set $\widehat{\mathcal{S}}_{f} \subseteq \mathcal{S}_{f}$ such that $\left|E_{S}(s)\right|=1$ for each $s \in \widehat{\mathcal{S}}_{f}$ and $\bigcup_{s \in \mathcal{S}_{f}} E_{S}(s)=\bigcup_{s \in \widehat{\mathcal{S}}_{f}} E_{S}(s)$. The optimal objective function value of problem $P\left(\gamma, \mathcal{S}_{f}\right)$ is equal to the optimal objective function value of problem $P\left(\gamma, \widehat{\mathcal{S}}_{f}\right)$.

Proof. Due to Proposition 1, the optimal objective function value of problem $P\left(\gamma, \widehat{\mathcal{S}}_{f}\right)$ is an upper bound on the optimal objective function value of problem $P\left(\gamma, \mathcal{S}_{f}\right)$. But it is also a lower bound since problem $P\left(\gamma, \widehat{\mathcal{S}}_{f}\right)$ is a relaxation of problem $P\left(\gamma, \mathcal{S}_{f}\right)$ (due to the fact that $\widehat{\mathcal{S}}_{f} \subseteq \mathcal{S}_{f}$ ).

Proposition 1 and Corollary 3 not only enable considering a limited set of network states, but also allow for simplifying constraints (3a), (3b), (3c), (3d), (3e), (3f), (3g), and (3h) defining set $Q_{Z}(c, x, \mathscr{F})$, due to the fact that only one link for each state needs to be analysed. First, since $|\mathscr{F}|=1$, the number of variables and constraints is heavily reduced, and index $e \in \mathscr{F}$ is actually not required. Second, as it can be noticed that it is not necessary to examine directly whether the considered link is saturated (the link must be saturated if at least one $Z_{d p}$ is less than 1 ), variables $u_{e}$ and constraints $(3 b)$ and $(3 c)$ are actually not required.

Still, problem (4a), (4b), (4c), and (4d) is nonlinear due to the nonlinearity of constraint (1d). However, if singlepath routing of demands is assumed, the problem can be formulated as a MIP. For each $d \in \mathscr{D}$ and $p \in \mathscr{P}(d)$, let $x_{d p}^{\prime}$ be a binary variable that equals 1 if the sessions of demand $d$ use path $p$ and 0 otherwise. Then variables $x_{d p}$ can be replaced with variables $x_{d p}^{\prime}$ using substitution $x_{d p} \equiv s(d) x_{d p}^{\prime}$. Finally, expression $X_{d p s} \equiv x_{d p}^{\prime} z_{d p s}$ can be linearised requiring that $0 \leq X_{d p s} \leq x_{d p}^{\prime}$ and $z_{d p s}-\left(1-x_{d p}^{\prime}\right) \leq X_{d p s} \leq z_{d p s}$.

Thus, problem $P\left(\gamma, \mathcal{S}_{f}\right)$ can be treated as a single-path routing MIP problem with additional constraints. And as analysed in $[5,7]$ the single-path routing problem can be solved quite efficiently. Hopefully, the additional constraints are not very demanding, as, in particular, the actual values of variables $z_{d p s}$ are not critical-it is only important if they are greater or equal to $\gamma$. However, the numerical experiments that are supposed to illustrate the computational complexity of the problem will be the subject of a separate paper.

\section{Conflict of Interests}

The author declares that there is no conflict of interests regarding the publication of this paper.

\section{Acknowledgment}

This work was supported by National Science Centre, Poland, under Grant 2011/01/B/ST7/02967.

\section{References}

[1] Y. Li, M. Pioro, and V. Angelakis, "Design of cellular backhaul topology using the FSO technology," in Proceedings of the 2nd International Workshop on Optical Wireless Communications (IWOW'13), pp. 6-10, Newcastle Upon Tyne, UK, October 2013.

[2] D. Borah, A. Boucouvalas, C. Davis, S. Hranilovic, and K. Yiannopoulos, "A review of communication-oriented optical wireless systems," EURASIP Journal on Wireless Communications and Networking, vol. 2012, article 91, 2012.

[3] M. Pióro and D. Medhi, Routing, Flow, and Capacity Design in Communication and Computer Networks, Morgan Kaufman, Boston, Mass, USA, 2004.

[4] W. Ogryczak, M. Pióro, and A. Tomaszewski, "Telecommunications network design and max-min optimization problem," Journal of Telecommunications and Information Technology, vol. 3, pp. 43-56, 2005.

[5] A. Tomaszewski, Optimisation of Telecommunications Transport Networks, Wydawnictwa Politechniki Warszawskiej, Warsaw, Poland, 2013.

[6] A. Tomaszewski, "A polynomial algorithm for solving a general max-min fairness problem," European Transactions on Telecommunications, vol. 16, no. 3, pp. 233-240, 2005.

[7] M. Pióro, A. Tomaszewski, M. Dzida, M. Mycek, and M. Zagożdżon, "Literature survey, models, polyhedral results, and exact and heuristic methods for shortestpath routing problems," Tech. Rep., Institute of Telecommunications, Warsaw University of Technology, Warsaw, Poland, 2007. 


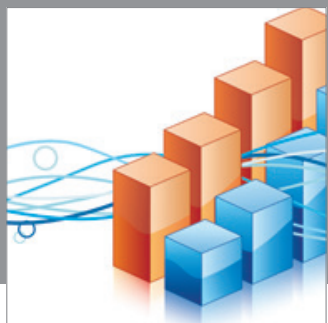

Advances in

Operations Research

mansans

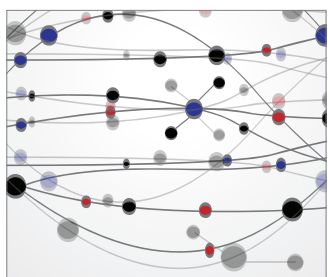

The Scientific World Journal
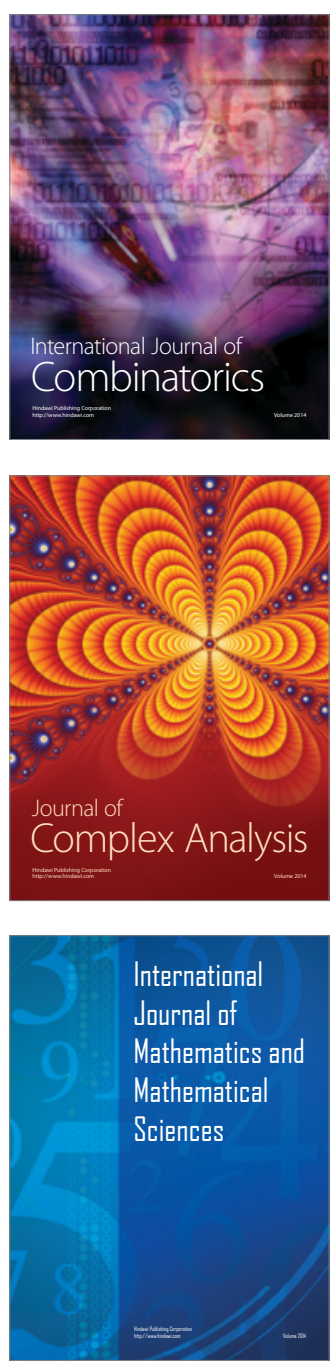
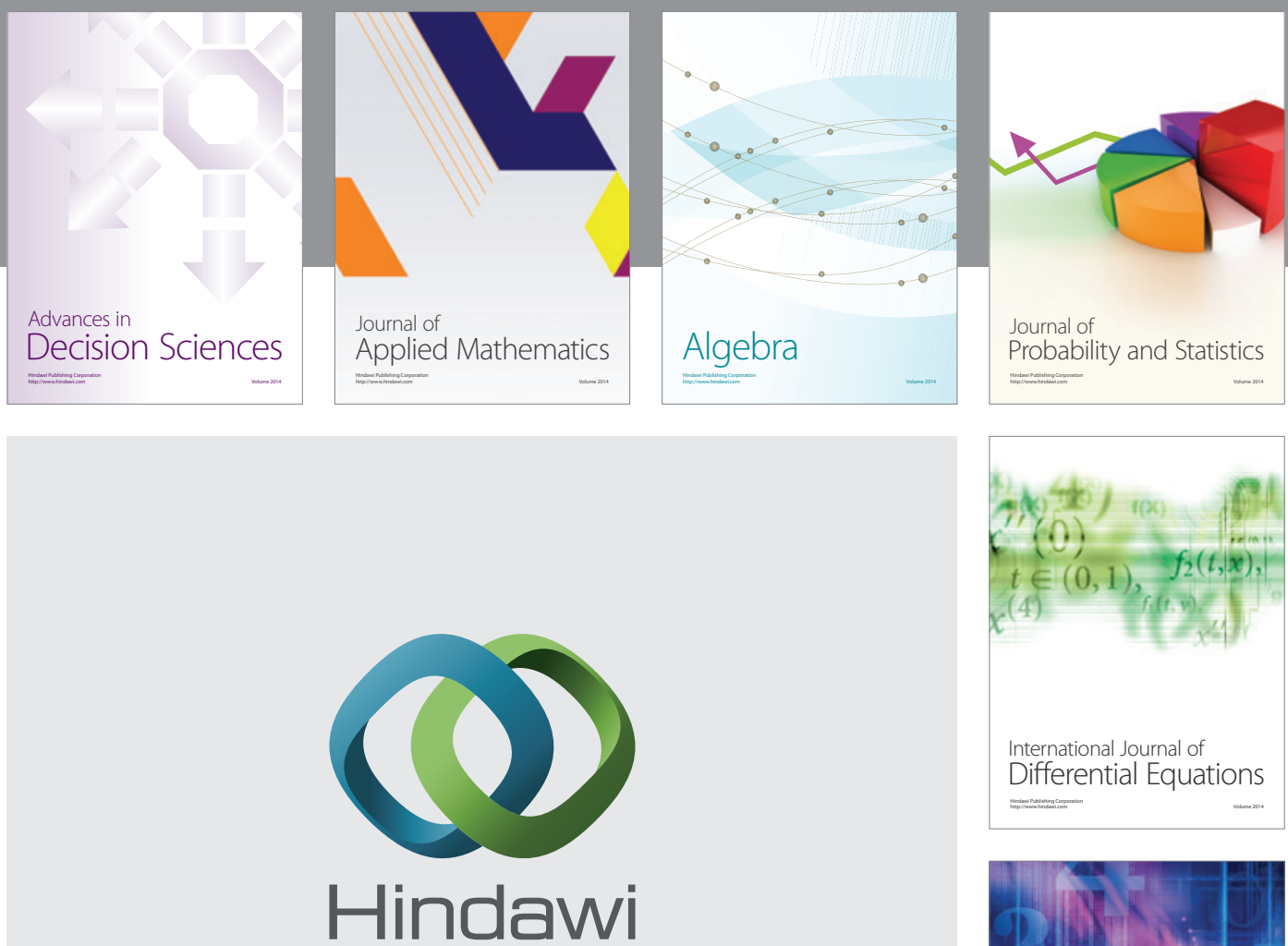

Submit your manuscripts at http://www.hindawi.com
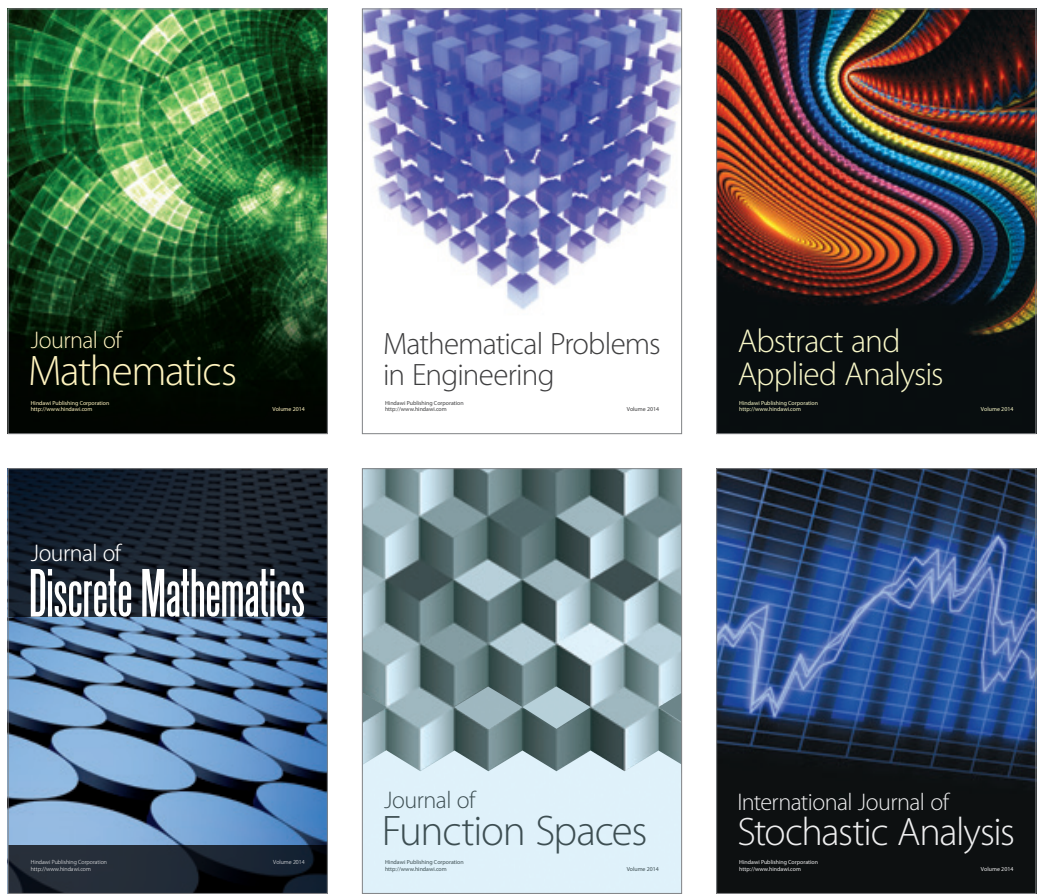

Journal of

Function Spaces

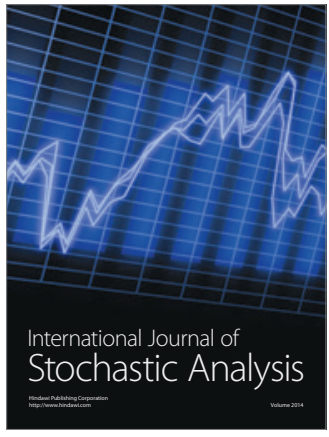

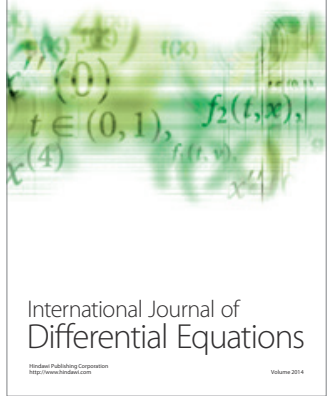
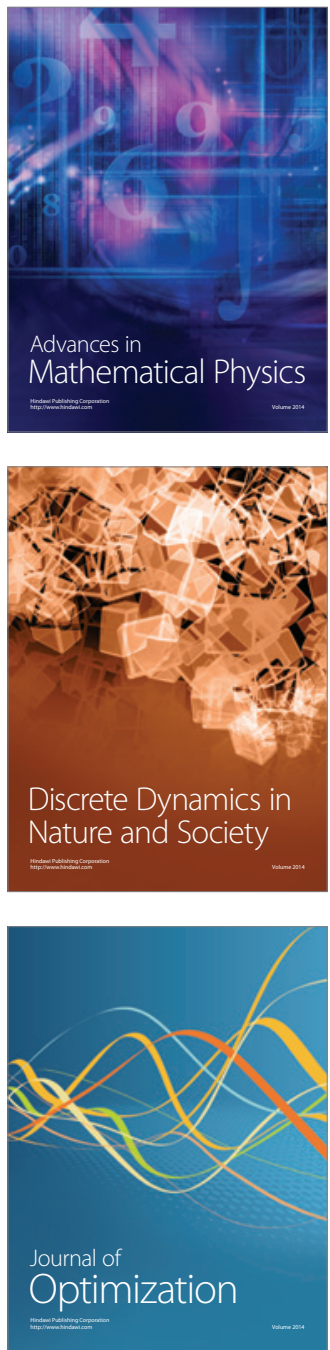